\title{
Personalised medicine - A recent revolution in periodontics
}

\author{
Gomathi G D ${ }^{1 *}$, S. Gopalakrishnan², Uma Sudhakar ${ }^{3}$ \\ ${ }^{1}$ Post Graduate Student, ${ }^{2}$ Professor, ${ }^{3}$ Professor and Head, Dept. of Periodontics, Thai Moogambigai Dental College \& Hospital, Tamil \\ Nadu, India
}

*Corresponding Author: Gomathi G D

Email: drgomathigd@gmail.com

\begin{abstract}
"Personalized medicine refers to applying pharmacogenomics to clinical management of an individual which utilizes new molecular tools \& technology to facilitate individualized health care based on person's unique characteristics enabling a higher level of personalization. Modifying genetic setup can prevent or cure a disease. Though the term personalized medicine Discovery of genes involved in drug metabolism \& drug alternatives can prevent adverse drug interaction. This article highlights about personalized medicine \& application of Pharmacogenomics in the field of Periodontology.
\end{abstract}

Keywords: Genetics, Proteomic, Pharmacogenomics.

\section{Introduction}

Not all patients respond similarly to a drug administered. This is the reason for developing the concept of personalized medicine. Though the term "Personalized medicine" sounds unique, it is not a new concept but an extended form of traditional approaches in understanding and treating disease with greater precision. It is a medical model involving customization of healthcare with medical decisions, practices, and/or products tailored according to the each patient need based. ${ }^{1}$ The older concept of 'one cut fits all' was replaced by newer personalized medicine concept because same treatment plan is not successful for all the patients. Inter-individual variability to treatment response develops as a consequence of host-environmentmicrobial interactions that give rise to a specific clinical phenotype. This phenotype is the reason for difference in patient's response to the given treatment. ${ }^{2}$ The field of personalized medicine relies on genetic, proteomic information and patient characteristics to individualize treatment. Patient's profile with genetic variations guides the selection of drugs or treatment protocols minimizing harmful side effects \& ensuring successful treatment outcomes. Periodontal diseases are complex, multifactorial inflammatory disease affecting the bone and soft tissue of the teeth. Genetics plays a major role in periodontitis. Though personalized medicine is an emerging field, the logic behind it is nothing new. Individualized care is provided based on a person's unique genetic profile that uses molecular tests like gene mapping, DNA profile, receptor gene amplification test, fluorescence insitu hybridization (FISH), microarray test, AmpliChip CYP450 Test, etc. ${ }^{3}$ Personalized medicine seeks to prevent and treat disease through analyzing the variables of every individual as "what is successful for one person may not work for someone possessing different genes, existing in a different environment, and a different lifestyle".

\section{History}

The observations of highly variable drug response, which began in the early $1950 \mathrm{~s}$, led to the birth of a new scientific discipline arising from the confluence of genetics, biochemistry, and pharmacology known as pharmacogenetics. $^{3}$ Sir Archibald Garrod [1902] was the first person to make connection between genetic inheritance and susceptibility to a disease called as Alkaptonuria. In 1956, the discovery of a genetic basis for selective toxicity was made for the antimalarial drug (primaquine). In 1977, discovery of role of cytochrome P450 metabolic enzymes in chemically altering levels in bloodstream led to the fact that variation in the level of these metabolic enzymes could have a definite impact on the dosage of a drug. In the late 1990s, the term 'Personalized medicine' was first introduced after its scientific basis had been set over the preceding decades. Pharmacogenomics combines genomics and pharmacology for developing effective, safe medications and doses based on a person's genetic makeup. ${ }^{5}$

Reasons for development of pharmacogenomics are:

1. The pathogenesis of most diseases are polygenic in nature

2. Most drugs exert actions in a multigenic manner

3. Complex interactions between the genes, environment, drugs, and pathogens

Pharmacogenomics is the study of the identification of genes and their corresponding products which influence genes and their corresponding products that influence individual variation in the efficacy and/or toxicity of individual variation and/or therapeutic products, and the application of genomic therapeutic products, and genomic information to help inform therapeutic product development and/or clinical application. ${ }^{6}$ This may include:

1. Choosing the most appropriate therapeutic product for a patient;

2. Selecting optimal dose;

3. Identifying those at risk for unexpected or more frequent adverse drug reactions.

\section{Using gene as a testing tool}

Genes are segments of DNA that are found in all cells that can influence a person's response to medications. Genes have various forms and different chemical messengers, interactions of which affect drug activity in the body. ${ }^{7}$ There 
are two types of gene variation. The first is single gene variation which is variation in the single gene that is sufficient to alter the phenotype (a mutation), and the second is complex interactions which involve mutations in many genes, often with small cumulative effects, and interaction with environmental factors. ${ }^{8}$ Lack of variation in genomic data is a well-recognized problem that creates challenges in identifying variants associated with disease and in diagnosing and treating patients. Ethnic differences run the risk of being misdiagnosed because of the lack of variations in genomic databases.

Using genome sequencing technology, these groups of individuals are analyzed for the presence and absence of a finite set of specific genetic variants across the genome through Genome-Wide Association Studies (GWAS). Different types of genetic tests are done to obtain and personalize an individual's treatment. Identifying genetic risk factors for serious Adverse Drug Reactions (ADRs) could decrease the costs of health care and improve patient outcomes. ${ }^{9}$ Identifying genetic variations (genotyping) in drug-metabolizing enzymes has led to physicians improving the dosing of drugs for different conditions. Genetic variation also plays a crucial role in drug-drug interactions. Genotyping provides an understanding of the genetic factors that may contribute to variability in drug response and can help maximize the likelihood of efficacious treatment and minimize ADRs. DNA sequence variations are described as mutations and as polymorphisms. Genetic mutations are deterministic of simple genetic diseases. ${ }^{2}$

Health care expenses can be reduced by analyzing full genome sequence of an individual, which aids in screening and tailoring the drugs and treatments thereby minimizing side effects and improving outcomes. Testing can also help to identify an individual's disease susceptibility. These functions all play a role in ensuring more targeted and costeffective health care into the future. ${ }^{10}$ DNA represents only static information about the state of a cell whereas transcriptomics and proteomics are capable of reflecting the dynamic state of molecular activity. For this reason it is likely that information about the DNA alone is not sufficient to accomplish the noble goals of personalized medicine. ${ }^{11}$

\section{Application}

The known applications of Pharmacogenomics include:

1. Improving drug safety

2. Reducing Adverse Drug Reactions

3. Tailoring treatments to meet patients' unique genetic predisposition

4. Identifying optimal dose;

5. Improving proof of principle for efficacy trials.

Pharmacogenomics can switch from reaction to prevention, direct selection of optimal therapy and reduce trial-and-error prescribing, avoiding adverse drug reactions, improving quality of treatment, alternative of routine medicines and reducing health care cost. Pharmacogenomics is applied in several areas of medicine, like Cardiology, Oncology, $\&$ Psychiatry. In Forensic medicine, pharmacogenomics can be used to determine the reason of death in drug-related death cases where no findings emerge with autopsy.

\section{Personalised analysis for disease identification}

Data mining has identified several relevant genes including those involved in the NFKB complex-mediated inflammation process, consistent with previous observations on the role of inflammation in periodontitis. Microarray technology may provide more accurate and reliable method of identifying periodontal disease subtypes through the characterization of differences in gene expression profiles. Genomic analysis enables the identification of microorganisms associated with periodontitis that were previously unable to be cultured in the lab. Thus, array methods may subsequently be used clinically to identify patients with pathogenic microbes in their oral cavity.

Proteomic analysis allows the identification of differential levels of protein expression unique to oral cavities with periodontitis. Proteins produced in response to bacteria-induced periodontitis can be detected and used in early diagnosis and prevention of disease progression. Proteomic studies have implicated abnormal levels of various immune-related proteins in the possible development of periodontitis. Exposure to various environmental factors such as cigarette smoke and certain microbial agents has also been shown to increase the risk for periodontitis by altering protein expression within the oral cavity. Coupled with the characterization of a patient's microbial profile, genomic and proteomic information will lead to more informed decision making with regard to the application of specific antibiotics, anti-inflammatory drugs, and appropriate restorative procedures. ${ }^{12}$

Risk factors like smoking, diabetes and certain genetic variations are associated with more severe periodontal disease. However in spite of this observation, it is usually assumed that all adults are at equal risk and similar type of preventive and interceptive care is provided. Because of which, individuals who are at higher risk for developing the disease are inadequately treated and a individuals who are at lower risk to develop the disease are getting over-treated. Because of missed opportunities to prevent disease in high risk individuals and unnecessary services rendered in low risk individuals, health care costs appears unsustainable.

\section{Personalised medicine in periodontal disease}

Periodontal disease is a multifactorial disease affecting supporting structure of the teeth. Microorganisms in the biofilm are the main etiologic factors lead for periodontitis. It is also highly associated with systemic inflammation resulting in an increased risk for chronic diseases such as cardiovascular diseases, diabetes, metabolic syndrome, pneumonia and rheumatoid arthritis. ${ }^{13}$ Periodontitis involves intrinsic systemic analysis including genetics, metabolomics, transcrpitomics, metagenomics, infectogenomics, immunogenomics which interact with epigenetics in environment stimuli. The host immunity, along with local and systemic factors, plays a major role in disease progression. In periodontitis, specific mutations 
have been identified to cause the genetic basis of the disease ${ }^{14}$ From genetic point of view, periodontitis arises as a result of single nucleotide polymorphisms (SNPs). Various studies explored role of SNPs in periodontitis. Results varied in distribution of SNPs between various ethnic groups. Differences in genes encoding the interleukins IL-1, IL-6, and IL -10, Fc receptors (Fc RIIa, Fc RIIIa and Fc RIIIb), TNF- $\alpha$, vitamin D receptor, CD14 and MMP-1, E-selectins, CD14, toll-like receptors were found..$^{15}$

William Giannobile ${ }^{16}$ utilized personalized medicine approach in his study in which he evaluated tooth loss that is one of the terminal outcome of untreated periodontal disease in 5117 periodontitis patients, over a period of 16year.Patients were stratified based on three risk factors, which were smoking, diabetes and interleukin-1 (IL-1) positive genotype. If patients had one or more of these risk factors, the investigators classified them as being at highrisk of periodontitis, and at low risk if they had none of the three risk factors. Authors found that, in low-risk patients, tooth loss was not impacted based on whether the patient visited the dentist once or twice annually. ${ }^{17}$ In high-risk patients, two preventive visits compared to one visit per year significantly reduced the number of patients who had tooth loss events.

Personalized medicine for periodontal diseases may soon involve utilization of saliva to develop subclinical profiles, identifying and measuring specific genotypes, phenotypes, putative pathogens, inflammatory markers and collagen-degradation biomarkers to make informed clinical decisions about disease susceptibility, site-specific risk and treatment interventions. When considering the possible utilization of saliva in a personalized model for periodontal disease, one can imagine implementation at multiple levels - screening, disease detection, monitoring of treatment outcomes and identification of refractory or progressing cases. During the screening phase, the use of saliva to identify patients at risk for future disease activity opens the door for heightened risk management strategies, preventive care and/or behavior change on the part of the patient to prevent the onset of disease. ${ }^{18}$ At the diagnostic stage, identifying the presence of disease at the earliest possible stage may allow for less invasive, less costly treatment. Saliva has a simple mechanism for monitoring treatment outcomes, as well as identification of refractory sites, additionally provides both the patient and the clinician with valuable information regarding the present state of the disease. ${ }^{19}$

Periodontitis can be prevented by stopping the progression of gingivitis through oral hygiene care. Periodontitis can be inhibited by activating STAT6 via over expression of 2 receptors of IL-4 which inhibits expression of IL-11 and Leukemia inhibitory factor. Release of anti inflammatory cytokine like IL-10 can resolve inflammation prevent bone destruction. Developing a single chair side test with multiple biomarkers can help in diagnosing the disease at early stage and helps in better treatment. ${ }^{20}$

Pharmacogenomics optimizes treatment, for developing more effective therapies, and improving patient outcomes. It is not clear at present whether data from one ethnic population can be extrapolated to another population.

\section{Goals of personalized medicine}

Personalized medicine mainly aims is to provide optimized medical care and better outcome for each individual. Bristow $^{21}$ described the goal of pharmacogenetic therapy to improve the probability of response and to reduce response heterogeneity, to increase the magnitude of response, to decrease the probability of adverse effects and/ or serious adverse effects, to improve the success rate of drug development, to reduce the cost of medical care and to provide additional marketing exclusivity. Based on molecular diagnosis and genetic makeup of each patients, optimal drug selection and dose adjustment is planned. Although not all drugs can be personalized, there is most likely to be clinical significance in tailored medicine for pro-drugs, drugs with an arrow therapeutic index and drugs that target a key molecule or a critical pathway. Drug safety is the main criteria from pharmacogenetics and pharmacogenomics that can benefit patients. Tumor responses to the inhibitors of oncogenic tyrosine kinase are associated with the presence of activating mutations within the genes encoding the target kinases; targeted cancer therapy is thus a promising individualized drug therapy. ${ }^{22}$

\section{Limitations of personalized medicine}

Limitations include the use of genotype- guided therapies that requires clinicians to have a level of knowledge sufficient to understand and interpret the rationale for prescribing for certain genotypes. ${ }^{23}$ Clinician acceptance may be a significant barrier to introduction of widespread genotype-based therapy. SNPs are very common in human genome; therefore millions of SNPs must be identified and analyzed to determine their involvement in drug response. Knowledge on genes that are involved with each drug response is limited. Since many genes are likely to influence responses, it is highly time-consuming and complicated to associate gene variations and drug response. ${ }^{24}$ Due to limited drug alternatives, patients carrying gene variations may be left without any alternatives for treatment. Also, the need for alternative drugs that serve only a small portion of the population and the great cost of bringing a new drug in market may be a disincentive for drug companies. Cost is a potential barrier to the widespread implementation of pharmacogenetic testing into routine clinical practice. Test information needs to be balanced against other clinical and cost considerations, and a modified rather than strict gatekeeper model may be appropriate. Drug developing expenses can be reduced through an efficient drug development process. ${ }^{25}$ In-vitro quantification of drug and drug metabolites in blood sample of a patient can be done through \liquid chromatography, mass spectrometery, flow cytometery and SPR biosensing. Using ELISA for applying personalized medicine in regular practice may not be really possible as they are expensive, time-consuming require specialized reagents and personnel. 


\section{Conclusion}

Understanding the disease pathways, genomic interactions, and novel biomarkers of oral conditions before the occurrence of the disease will help in preventing the disease and, to some extent, will guide treatment planning. Prompt diagnosis of patients can help in getting benefit from targeted therapies. Further longitudinal studies could help in understanding personalized medicine better. It is necessary to bring awareness about Pharmacogenomics to every individual and their influence in drug response. Incorporation of the pharmacogenomic data into clinical practice is a challenge for the future.

\section{Source of funding}

None.

\section{Conflict of interest}

None.

\section{References}

1. Geoffrey S. Ginsburg. Genomic and personalized medicine: foundations and applications. Pharmacogenomics 2009;154: 277-87.

2. Evans WE, McLeod HL. Pharmacogenomics - drug disposition, drug targets, and side effects. New Engl J Med 2003;348:538-49.

3. Kristine R. Crews. Pharmacogenomics and individualized medicine: Translating science into practice. Clin Pharmacol Ther 2012;92:467-75.

4. Chan IS, Ginsburg GS. Personalized medicine: progress and promise. Ann Rev Genomics Hum Genet 2011;12:217-44.

5. Hamburg MA \& Collins FS. The path to personalized medicine. New Engl J Med 2010;363:301-4

6. D Gurwitz, JE Lunshof, G Dedoussis, CS Flordellis, U Fuhr, J Kirchheiner et al. Pharmacogenomics Education: International Society of Pharmacogenomics. Pharmacogenomics Journal 2005; 5: 221-225.

7. Laviero Mancinelli. Pharmacogenomics: The promise of personalized medicine. AAPS Pharm Sci 2000;2:29-41.

8. Shastry BS. Pharmacogenomics and concept of individualized medicine. J Pharmacogenomics 2006;6:16-21.

9. Piquer-Miller M \& Grant DM. The art and science of personalized medicine. Clin Pharmacol Ther 2007;81:311-5.

10. Helgadottir A, Thorleifsson G, Manolescu A, Gretarsdottir S, Blondal T, Jonasdottir A et al. A common variant on chromosome 9p21 affects the risk of myocardial infarction. Sci 2007;316:1491-3.

11. Tester DJ, Ackerman MJ. Genetic testing for potentially lethal, highly treatable inherited cardiomyopathies/channelopathies in clinical practice. Circ 2011;123:1021-37.

12. Oates TW, Graves DT, Cochran DL .Clinical, radiographic and biochemical assessment of IL-1/TNF-alpha antagonist inhibition of bone loss in experimental periodontitis. J Clin Periodontol 2002;29:137-43.

13. F. D'Aiuto, M. Parkar, G. Andreou et al. Periodontitis and systemic inflammation: control of the local infection is associated with a reduction in serum inflammatory markers. $J$ Dent Res 2004;83:156-60.

14. Kinane DF, Hart TC. Genes and gene polymorphisms associated with periodontal disease. Crit Rev Oral Biol Med 2003;14(6):430-49.

15. . Nadeem Y, Karimbux NY, Saraiya VM, Elangovan S, et al. Gene polymorphisms and chronic periodontitis in adult whites: a systematic review and meta-analysis. $J$ Periodontol 2012;83(11):1407-19.

16. Giannobile WV, Braun TM, Caplis AK, Doucette-Stamm L, Duff GW, Kornman KS. Patient stratification for preventive care in dentistry. J Dent Res 2013;92:694-701.

17. S. Podzimek, J. Mysak, T. Janatova, J. Duskova. Creactive protein in peripheral blood of patients with chronic and aggressive periodontitis, gingivitis, and gingival recessions. Mediators Inflamm 2015;7:234-41.

18. Kinney JS, Ramseier CA, Giannobile WV Oral fluid-based biomarkers of alveolar bone loss in periodontitis. Ann NY Acad Sci 2007;1098:230-51.

19. J.A. Loo, W. Yan, P. Ramachandran, D.T. Wong. Comparative Human Salivary and Plasma Proteomes. J Dent Res 2010;89:1016-23.

20. Engebretson SP, Grbic JT, Singer R, Lamster IB. GCF IL1 beta profiles in periodontal disease. J Clin Periodontol 2002;29:48-53.

21. Bristow M. New Frontiers in Personalized Medicine: Cardiovascular Research and Clinical Care. Personalized Medicine Coalition. Washington DC: The George Washington University; January 6, 2011. Pers Med Dent J Orofacial Sci 2017;9:3-6.

22. Brazell C, Freeman A, Mosteller M. Maximizing the value of medicines by including pharmacogenetic research in drug development and surveillance. Br J Clin Pharmacol 2002;53:224-31.

23. Pavo Filaković \& Anamarija Petek. Personalised pharmacotherapy in psychiatry. $J$ Psychiatry Danub 2009;21:341-6.

24. Lazarou J, Pomeranz BH, Corey PN. Incidence of adverse drug reactions in hospitalized patients: a meta-analysis of prospective studies. JAMA 1998;279:1200-5.

25. Veenstra DL, Higashi MK, Phillips KA. Assessing the costeffectiveness of pharmacogenomics. AAPS Pharmsci 2000;2:E29.

How to cite this article: Gomathi G D, Gopalakrishnan S, Sudhakar U. Personalised medicine - A recent revolution in periodontics. Int J Periodontol Implantol 2019;4(3):69- 